UCRL-JC-128440

PREPRINT

\title{
Developments in Limited Data Image Reconstruction Techniques for Ultrahigh-Resolution X-ray Tomographic Imaging of Microchips
}

\author{
W. S. Haddad \\ J. E. Trebes
}

This paper was prepared for submittal to the

SPIE 1997 International Symposium on Optical Science, Engineering, and Instrumentation

San Diego, CA

July 27-August 1, 1997

August 20, 1997

This is a preprint of a paper intended for publication in a journal or proceedings. Since changes may be made before publication, this preprint is made available with the understanding that it will not be cited or reproduced without the permission of the author. 


\section{DISCLAMER}

This document was prepared as an account of work sponsored by an agency of the United States Government. Neither the United States Government nor the University of California nor any of their employees, makes any warranty, express or implied, or assumes any legal liability or responsibility for the accuracy, completeness, or usefulness of any information, apparatus, product, or process disclosed, or represents that its use would not infringe privately owned rights. Reference herein to any specific commercial product, process, or service by trade name, tradernark, manufacturer, or otherwise, does not necessarily constitute or imply its endorsement, recommendation, or favoring by the United States Government or the University of California. The views and opinions of authors expressed herein do not necessarily state or reflect those of the United States Government or the University of California, and shall not be used for advertising or product endorsement purposes. 


\title{
Developments in Limited Data Image Reconstruction Techniques for Ultrahigh-Resolution X-ray Tomographic Imaging of Microchips
}

\author{
W. S. Haddad and J. E. Trebes \\ University of California, Lawrence Livermore National Laboratory \\ P.O. Box 808, Livermore, CA 94551
}

\begin{abstract}
The use of soft $\mathrm{x}$-ray $(-1.8 \mathrm{KeV})$ nanotomography techniques for the evaluation and failure mode analysis of microchips was investigated. Realistic numerical simulations of the imaging process were performed and a specialized approach to image reconstruction from limited projection data was devised. Prior knowledge of the structure and its component materials was used to eliminate artifacts in the reconstructed images so that defects and deviations from the original design could be visualized. Simulated data sets were generated with a total of 21 projections over three different angular ranges: -50 to +50 , 80 to +80 and -90 to +90 degrees. In addition, a low level of illumination was assumed. It was shown that sub-micron defects within one cell of a microchip $\left(<10 \mu \mathrm{m}^{3}\right)$ could be imaged in 3-D using such an approach.
\end{abstract}

Keywords: $x$-ray tomography, image reconstruction, 3-D imaging, microchips, zone plates, iterative optimization

\section{INTRODUCTION}

A critical part of the development of semiconductor microcircuits (microchips) is the analysis and testing of existing products and newly fabricated prototype microchips. It is important for chip manufacturers to be able to accurately check what their microfabrication processes have made, and to examine exactly how and where microcircuits fail after a period of use. This information is critical to the development of smaller, faster and better microcircuits, and can lead to increased yield of "good" chips.

Ideally, chip manufacturers would like to be able to know accurately how their microstructures differ from the specified design and be able to "see" the exact structure of defects that exist in the microcircuit. Currently they can determine the location of a defect to within one "cell" of the circuit by means of electrical tests, however, to determine the exact morphology of the defect, present practice is to etch away the bulk material surrounding the conductors and examine the metallic structures using scanning electron microscopy (SEM) 1,2 . This process is costly tedious and can take several days, and therefore allows the examination of a very limited number of samples. In addition, there is the possibility of damage occurring to the structures of interest during the etching and imaging process, thereby bringing into question the accuracy of the determinations.

Based on past experience with ultrahigh-resolution soft $x$-ray tomography $3-5$, we generated, and tested through numerical simulations, a conceptual design for a system capable of producing 3-D images with good contrast for features of interest within one cell $\left(<10 \mu \mathrm{m}^{3}\right)$ of a standard microchip, and with resolutions in the range of $100 \mathrm{~nm}$. Limited data imaging with soft $x$-rays could allow the information to be collected with greatly reduced sample preparation requirements, and reduce the chances of damage to the sample during the evaluation process. The imaging concept is a type of high resolution tomography similar to that described elsewhere ${ }^{6,7}$ but fine tuned for 3-D imaging of microchips.

\section{IMAGING SYSTEM AND METHODS}

A schematic of an imaging system suitable for $\mathrm{x}$-ray nanotomography of microchips is depicted in Figure 1 . Monochromatic $x$-rays from the source are collected and concentrated onto the ROI by a condenser optic. This optic could in principle be a multilayer coated toroidal or elliptical mirror, a Kirkpatrick-Baez mirror system ${ }^{8}$ or a zone plate. The sample is mounted on a single axis rotation stage, and positioned such that the ROI is as close to being centered on the rotation axis as possible. A fresnel zone plate is used to form a magnified image of the ROI directly onto a digital $x$-ray sensitive array detector (CCD 
based). A slice of the ROI is indicated in the figure. 3-D reconstructions are done by reconstructing individual slices and stacking them to form the full image.

Hard $\mathrm{x}$-ray zone plates are currently available for the $\mathrm{x}$-ray energy range of 1 to $4 \mathrm{KeV}$ and have a finest zone width of $\sim 2000$ $\AA$. These zone plates are fabricated by $x$-ray lithography and are similar to those described elsewhere 9 . When used as focusing elements, these zone plates can produce near diffraction limited spot with a radius equal to 1.22 times the finest zone width ${ }^{10}$. When used in imaging mode these optics can produce images with a transverse resolution of $2000 \AA$ to 2500 $\AA$. It is expected that zone plates with finest zone widths of $750 \AA$ will be available by 1998 . For the imaging system described in this article, we will assume the use of zone plates having $-800 \AA$ finest zones and a corresponding point spread function (PSF) with a full width at half maximum (FWHM) of $\sim 1000 \AA$.

An outline of the overall imaging and analysis approach taken is as follows:

1. identify the region of interest (ROI) of the microchip via electrical testing methods

2. form an ideal numerical model of the ROI of the chip

3. record projections of the sample (radiographs) from a series of different angles

4. reconstruct a 3-D image of the ROI

a. use as much prior knowledge of the chip structure as possible

b. use iterative optimization methods for image reconstruction

c. reconstruct deviations from the design only

5. superimpose or highlight the defects on an image of the ideal model of the ROI for interpretation by the investigators

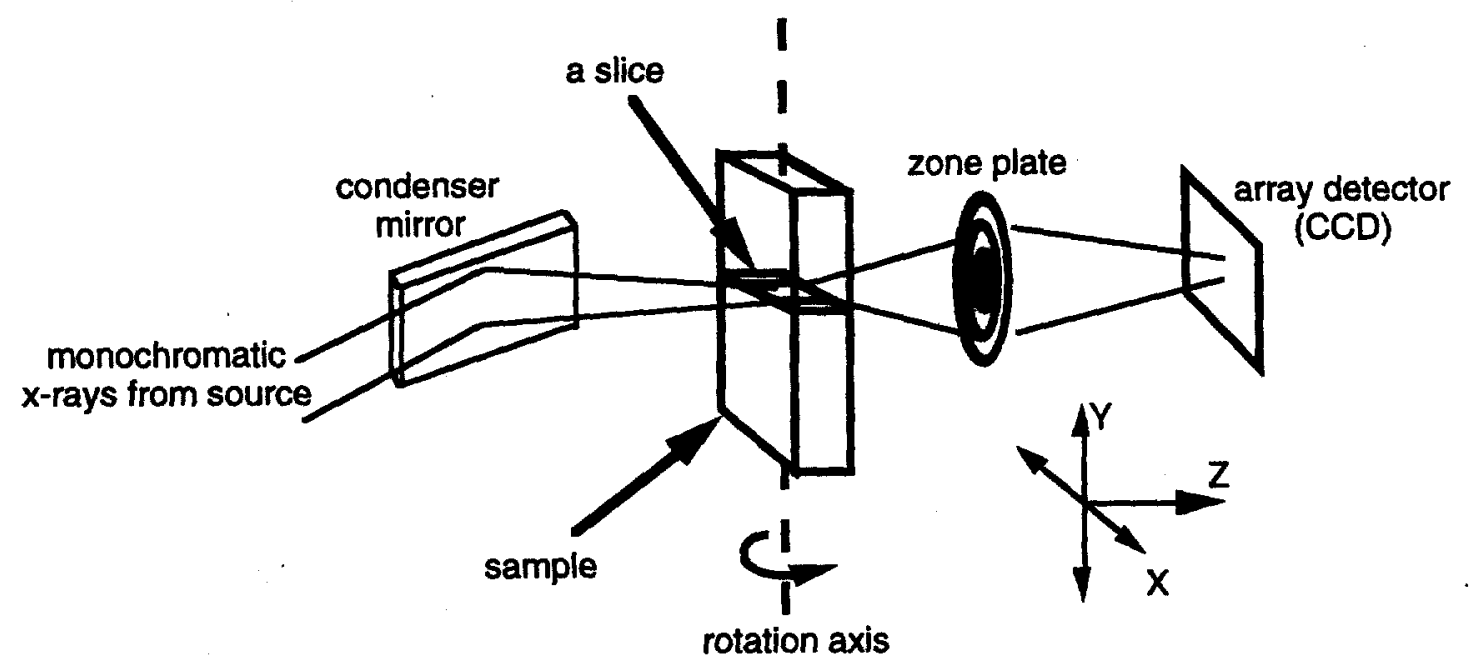

Figure 1. Schematic of an imaging system for $x$-ray nanotomography of microchips

\section{SIMULATIONS}

The primary construction materials used in current state-of-the-art microchips are silicon ( $\mathrm{Si}$ ) substrate material with $\mathrm{SiO}_{2}$ bulk material in the active region of the chip. The conductors in planes parallel to the substrate surface formed by lithography are made of aluminum (Al) with a thin capping layer of titanium (Ti) on both sides, and the interconnects between layers of conductors are made with tungsten (W) "plugs" or "vias". The primary difficulty in imaging the internal structure of the microchip comes in getting sufficient contrast between the $\mathrm{SiO}_{2}$ and the $\mathrm{Al}$ (and $\mathrm{Ti}$ ) conductors, while not suffering from occlusion caused by the highly absorbing $W$ vias. From tables of $x$-ray properties of materials we determined that there exists a maximum in absorption contrast between $\mathrm{Al}$ and $\mathrm{SiO}_{2}$ for a photon energy of $1.8 \mathrm{KeV}{ }^{11}$. 
For our simulations we constructed models of a single slice of the ROI of a hypothetical microcircuit. Since the properties of, and procedures for imaging each slice are the same, all the necessary information about performance of the system can be gotten by simulating the imaging process for a single slice. Two models were created for the simulations: an ideal model of the perfect structure as specified by the engineering drawings; and a realistic model of the same structure that would result from fabrication process including typical (but acceptable) deviations from the design, plus a range of defects which can also occur. These slice models are shown in Figures $2 a$ and $2 b$. The difference image, representing the differences between the ideal model of the ROI and the ROI containing defects, is shown in Figure 2c. The difference image can be separated into two sub-images: the positive difference image which represents regions of the chip where unwanted material is present, and the negative difference image which shows regions of the chip where material should be present, but is missing. The positive and negative difference images for our model are shown in Figures $2 \mathrm{~d}$ and $2 \mathrm{e}$ respectively. Table 1 contains a listing of defects and sources of noise pertinent to microchip imaging with soft $x$-rays, and delineates those which were and were not

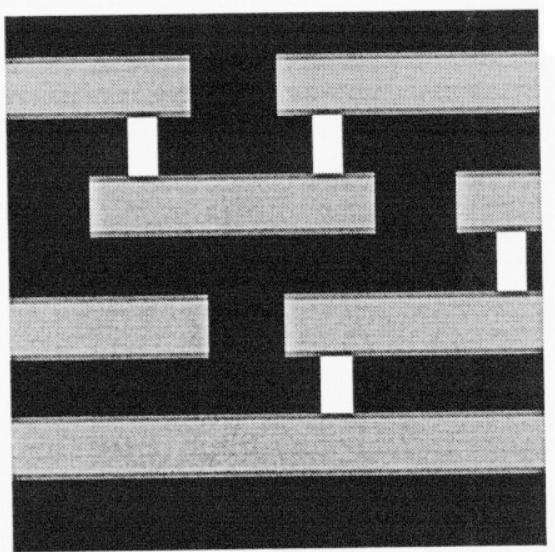

(a)

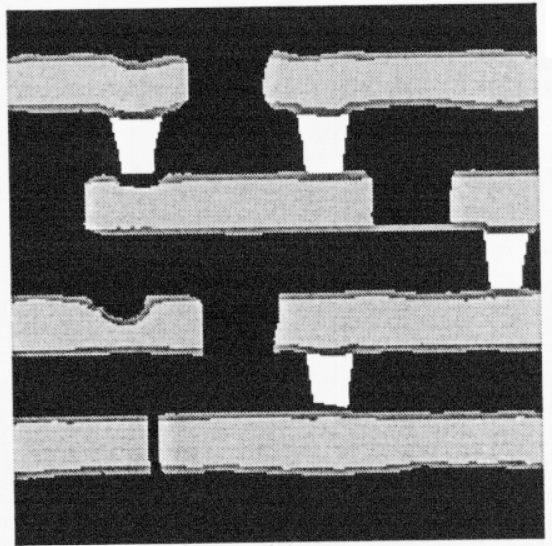

(b)

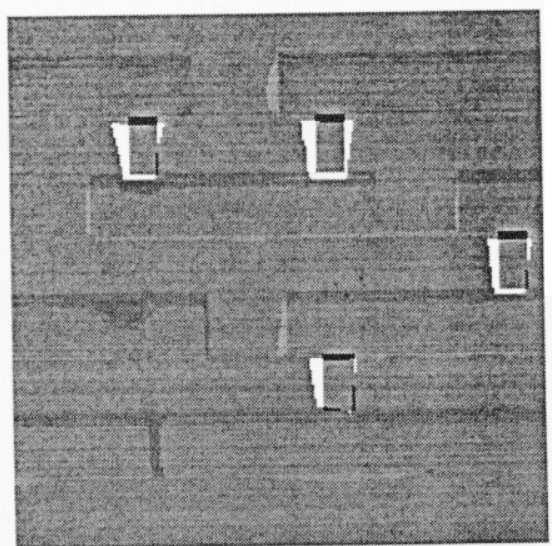

(c)

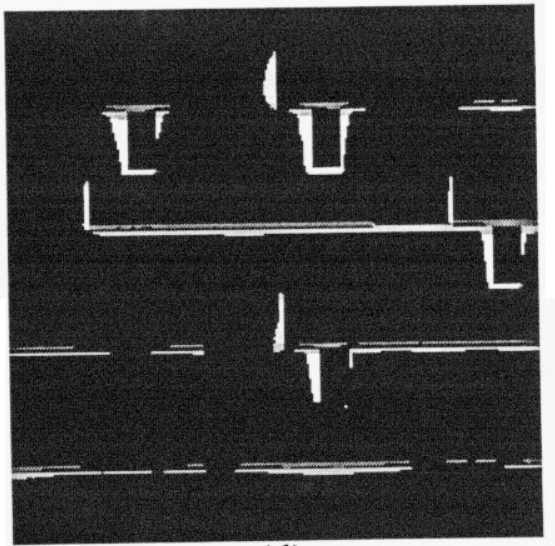

(d)

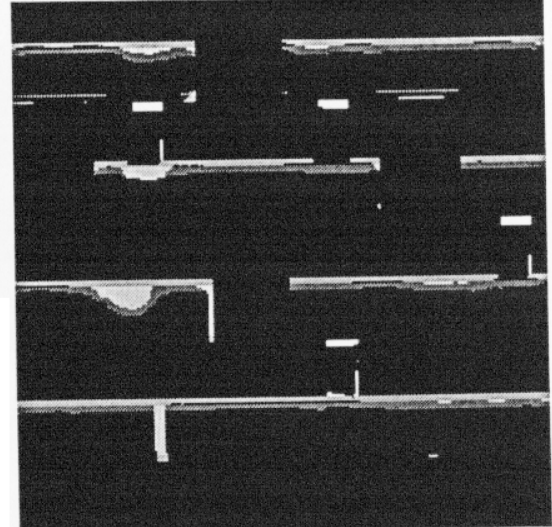

(e)

Figure 2. Numerical models of a microchip slice (crossection). In these images, brighter features represent higher values of the absorption coefficient $\alpha$, however, the look-up tables have been set so as to make all features clearly visible, and therefore the brightness is not proportional to the absorption coefficient. (a) Ideal model, (b) model with defects and deviations from the design, (c) difference image $=($ b) $-(a)$, (d) positive difference image $=l(c)>0 l$, (e) negative difference image $=|(c)<0|$. In (a) and (b), the black background is $\mathrm{SiO}_{2}$, bright wide gray bars are $\mathrm{Al}$ conductors, the thin layers of dark gray are $\mathrm{Ti}$ capping and the bright white bars are the $\mathrm{W}$ vias. 


\begin{tabular}{|c|c|c|c|}
\hline \multicolumn{2}{|c|}{ Defects } & \multicolumn{2}{c|}{ "Noise Sources" } \\
\hline included & not included & included & not included \\
\hline $\begin{array}{c}1000 \AA \text { short } \\
\text { (horizontal) }\end{array}$ & Cu inclusions & photon statistics & $\begin{array}{c}\text { misalignment } \\
\text { (rotational) }\end{array}$ \\
$\begin{array}{c}2000 \AA \text { open } \\
\text { (vertical) } \\
\text { "mouse bite" }\end{array}$ & $\begin{array}{c}\text { other material impurities } \\
\text { blurring due to optics } \\
\text { voids in SiO2 }\end{array}$ & $\begin{array}{c}\text { misalignment } \\
\text { (transverse) } \\
\text { angles }\end{array}$ & $\begin{array}{c}\text { inaccuracies in projection } \\
\text { angles }\end{array}$ \\
$1000 \AA$ W open & $\begin{array}{c}\text { limited angular range of } \\
\text { projections } \\
\text { limited number of } \\
\text { projections }\end{array}$ & $\begin{array}{c}\text { non-uniform illumination of } \\
\text { the sample } \\
\text { non-compact support } \\
\text { electron-migration }\end{array}$ \\
lack of flatness & & & x-ray scattering \\
\hline
\end{tabular}

Table 1. List of defects in the numerical models used and sources of noise in the imaging simulations which were and were not included in the simulations.

All the simulations were done assuming $1.8 \mathrm{KeV} \mathrm{x}$-rays because according to the Henke data, there is maximum contrast between $\mathrm{Al}$ and $\mathrm{SiO}_{2}$ at this photon energy. Modeling of the absorption was done according to Beer's law which is given in Equation. 1 and represents transmission through a series of $n$ different materials of thickness $x_{i}$. The absorption coefficients, $\alpha_{\mathrm{i}}$, were obtained using a computer program called XCal that interpolates the Henke data in order to calculate absorptions for elements and compounds. The value of each pixel in the slice models was chosen as the product the absorption coefficient, $\alpha_{i}$, for the material with the pixel dimension, $\delta$. Thus the value, $p$, of each pixel in a projection of the slice will be $p=\Sigma \alpha_{i} \delta$ along a ray through the slice, and the total transmission at each pixel in a projection can then be calculated as $\mathrm{I}=\mathrm{I}_{0} \mathrm{e} P$.

$$
I=I_{0} \exp \left[-\left(\alpha_{1} x_{1}+\alpha_{2} x_{2}+\ldots+\alpha_{n} x_{n}\right)\right]
$$

Since we were assuming that the zone plate would have a PSF with a FWHM of $1000 \AA$, we chose a characteristic pixel dimension, $\delta$, of $350 \AA$ in order to have spatial oversampling of -3 . This also gave an appropriate field of view of $\sim 9 \mu \mathrm{m}^{2}$ with $256 \times 256$ pixels per slice. Table 2 lists the pixel values, $\alpha \delta \delta$, for all of the materials used in the modeling. Note that the absorption coefficient for $\mathrm{W}$ is nearly an order of magnitude greater than that of $\mathrm{Al}$ at $1.8 \mathrm{KeV}$. The strong relative absorption of $\mathrm{W}$ is problematic for tomographic imaging of microchips, and will require special techniques in cases where limited data is available. We assumed a very low incident light level of $10^{4}$ photons per projection pixel before passing through the ROI. Simulated projection data sets were obtained using the model slices in Figure $2 a$ and $2 b$, obtaining projections by calculating $p=\Sigma \alpha \alpha_{j} \delta$ for each pixel in each projection, converting the values of $p$ to $I$ using $I=I_{0} e P$, convolving each projection with the PSF of the zone plate to include blurring due to the imaging optic, converting I into number of photons, $N$, by integerizing and adding gaussian distributed random noise with a standard deviation $N^{1 / 2}$. 


$$
\delta=350 \AA \text {, photon energy }=1.8 \mathrm{KeV}
$$

Material Pixel Value $\left(\alpha_{\mathrm{i}} \delta\right)$

$\begin{array}{cc}\mathrm{W} & 0.159534 \\ \mathrm{Al} & 0.027855 \\ \mathrm{Ti} & 0.020505 \\ \mathrm{SiO}_{2} & 0.006174 \\ \mathrm{Si} & 0.002522\end{array}$

Table 2. List of the pixel values, $\alpha_{i} \delta$, for all of the materials used in the microchip slice models shown in Figure 2.

Limitations on the available data include both the number of projections as well as the angular range over which the projections can be recorded. For microchip imaging the primary factor in limiting the number of projections would most likely be time and source brightness, while the limiting factor on the angular range would be due to the physical extent of the sample. In this case, the sample is assumed to be a thin flat section of the microchip produced by removing all the extraneous packaging and borders needed for electrical connections, and etching away most (but probably not all) of the Si substrate material. Limitation in the maximum angle at which projections can be recorded can come as a result of two things: as the sample is rotated so that rays pass through it at increasing incident angles, the total path they have to travel through the sample increases as $t / \cos (\theta)$, where $t$ is the thickness of the sample and $\theta$ is the incident angle. At some point, the ray paths through the material will become so long that too few photons will be recorded to make an acceptable projection. The zone plate will have to be placed within a few $\mathrm{mm}$ of the sample surface, therefore the absolute limit on the maximum angle comes when the sample physically touches the zone plate.

All the simulations were done assuming a total of 21 projections uniformly separated over three different angular ranges: $-50^{\circ}$ to $+50^{\circ},-80^{\circ}$ to $+80^{\circ}$ and $-90^{\circ}$ to $+90^{\circ}$. In all cases, an iterative optimization method known as Algebraic Reconstruction Technique (ART) was the basis of the algorithm used to form the images 12 .

Since a linear transformation exists between direct space and Radon space (the space spanned by the set of projections of the object, subtraction of the ideal model from the actual projection data can be done in Radon space prior to reconstruction. The difference projections can then be separated into positive and negative components and reconstructed separately to produce positive and negative difference images respectively. In cases with limited data, this approach holds certain advantages. Sparse, high contrast objects are always easier to reconstruct, and iterative optimization methods for image reconstruction, such as ART, perform better on such data sets. Also, since the positive and negative components have been separated, the positivity constraint can be applied. This tends to reduce artifacts and noise in the image and helps iterative image reconstruction methods converge more rapidly.

As mentioned above, the primary difficulty with limited data imaging of microchips arises from the presence of highly absorbing $\mathrm{W}$ amidst the small, low contrast features of interest ( $\mathrm{Al}$ and $\mathrm{Ti}$ within $\mathrm{SiO}_{2}$ ). Figure 3 demonstrates this by showing standard ART reconstructions of the positive and negative difference images from 21 views for the $-50^{\circ}$ to $+50^{\circ}$ angular range case including shot noise and blurring due to the PSF of the zone plate. Significant artifacts are present in the images, particularly in the negative difference image (Figure $3 b$ ), and it is unclear which features represent real defects in the microchip. 


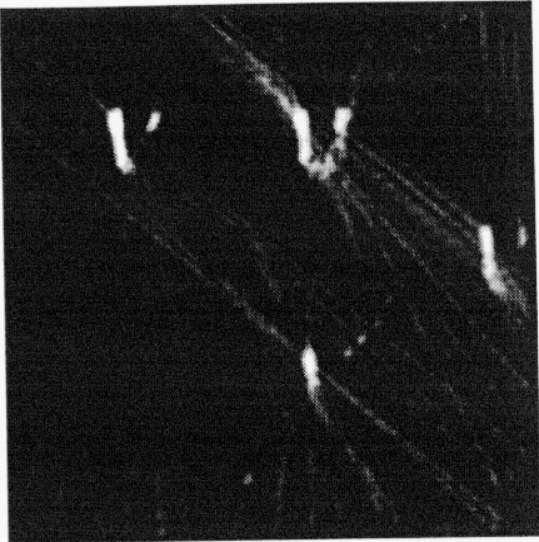

(a)

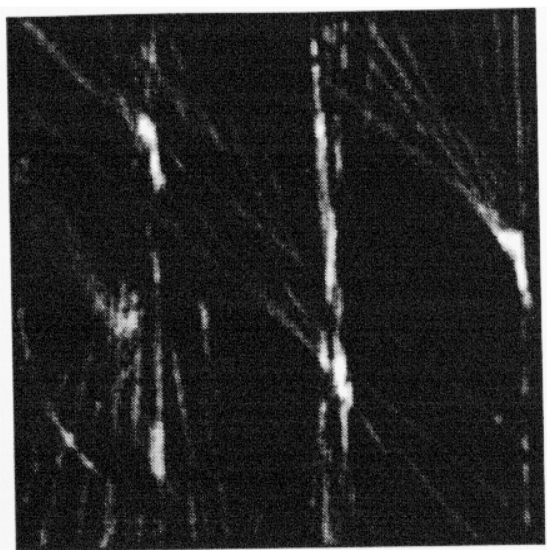

(b)

Figure 3. Standard ART reconstructions of (a) the positive and (b) the negative difference images from 21 views for the $-50^{\circ}$ to $+50^{\circ}$ angular range case including shot noise and blurring due to the PSF of the zone plate.

\section{RECONSTRUCTION ALGORITHM}

In order to deal with this problem, a specialized algorithm was developed. The approach taken was to effectively remove the W features from the difference projection data sets by subtraction in Radon space. Since we are removing all of the "correct", and therefore uninteresting features by subtracting out the ideal model of the microchip in Radon space, all that was needed was to adjust the ideal model so that it contains the actual W structures present in the real sample instead of the idealized W structures. Since there is a large difference in contrast between $W$ and everything else in the sample, it was possible to form a difference image of only the $\mathrm{W}$ defects as a first step in the overall imaging process. To do this, we designed an iterative technique which forms difference images of the sample using ART and then filters out everything which is known to be other than $\mathrm{W}$ on each iteration. The filtering can be done by making extensive use of prior knowledge about the structure including the known values for the absorption coefficients and the designated location of the W vias. Table 3 lists the types of prior knowledge used and its method of use in the filtering process. The result is an image of just the W defects which can then be added to the ideal model to produce a new model image whose projections can now be subtracted from the projections of the test sample. This greatly suppresses the adverse effects on the difference images of low contrast $\mathrm{Al}$ and Ti due to the presence of highly absorbing $\mathrm{W}$ in the sample.

design geometry

original design

regions of interest

material properties minimum absorption

maximum absorption

optical properties

noise statistics

PSF of zone plate

\author{
subtraction \\ masks
}

thresholding

clipping

$3 \times 3$ pixel smoothing kernel convolution with PSF before subtraction

Table 3. List of the types of prior knowledge used and corresponding methods of use for each in processing the reconstructions 
The following is an outline of the operations which compose the iterative W removal method:

1. begin with model of microchip and real projection data from sample

2. set working model $=$ ideal model

3. calculate projections of working model

- same projection angles as taken of sample

4. process projections of working model

- convolve with PSF of zone plate

5. subtract ideal projections from real projections

6. separate result into positive and negative parts

7. reconstruct positive and negative difference images using ART

8. process image to remove unphysical features

- apply $3 \times 3$ smoothing kernel

9. apply non-linear processing to pass only $\mathrm{W}$ defects

- thresholding: removes features of low absorption

- clipping: clamps high pixel values to aid for W

- masking: passes only features that lie between the $\mathrm{Al}$ conductors

10. produce new working model with reconstructed $W$ defects

- add positive W defect image to working model

- subtract negative $\mathrm{W}$ defect image to working model

11. if number of iterations $=\mathrm{N}$ (specified by user), then exit

12. else, goto 3

Figure 4 compares the ideal model with the model image to be subtracted in Radon space as it is modified to include the W defects over several iterations. Very few iterations were needed to produce a significant improvement in the final result. The positive and negative difference images of the $\mathrm{Al}$ and $\mathrm{Ti}$ features, plus residual $\mathrm{W}$ features, are shown in Figure 5 for ART reconstructions with subtraction of the projections of the modified ideal model produced by using the iterative $\mathrm{W}$ removal method described above for the $-50^{\circ}$ to $+50^{\circ}$ case. These images of the defects are significantly improved over those shown in Figure 3 which were produced without W removal. Clearly, some important features of interest are lost, however those that are present are real defects, and there is very little artifact or clutter in the images produced with iterative $\mathrm{W}$ removal. The final composite images of the ideal model with the defects superimposed are presented in Figure 6 for all three angular ranges, $-50^{\circ}$ to $+50^{\circ},-80^{\circ}$ to $+80^{\circ}$ and $-90^{\circ}$ to $+90^{\circ}$. From the images we estimated the size of the resolution element for the case of 21 projections and an angular range of the projections of $-50^{\circ}$ to $+50^{\circ}$. This is shown in Figure 7 .

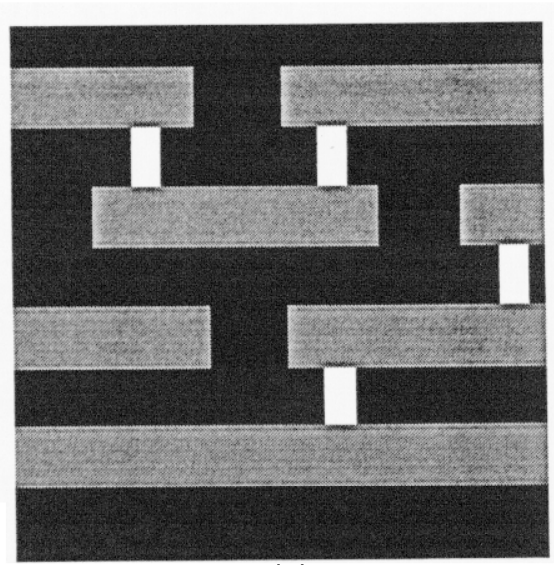

(a)

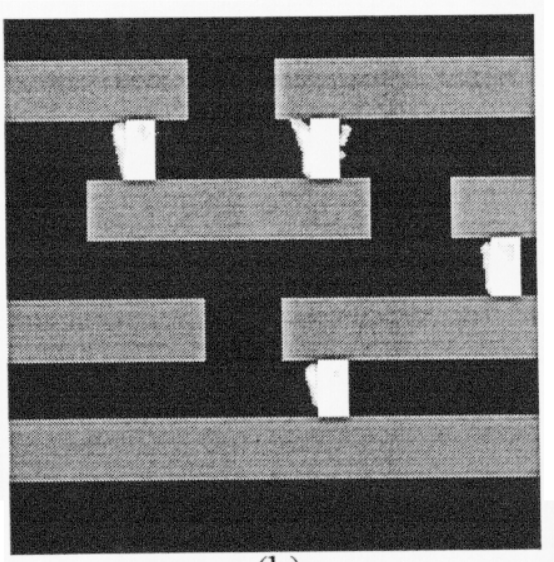

(b)

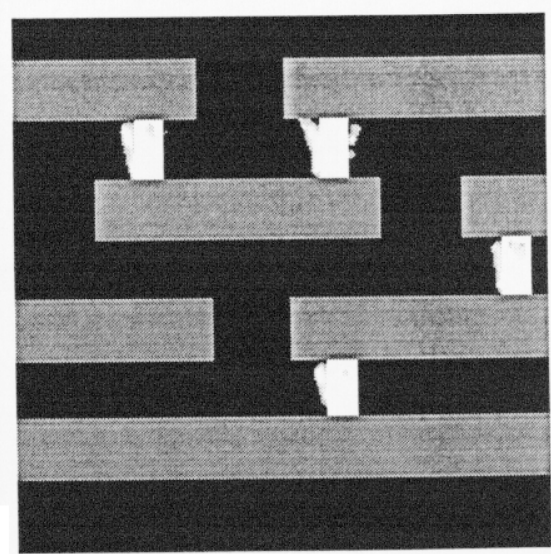

(c)

Figure 4. comparison of the ideal model (a) with the modified model image after two iterations (b) and five iterations (c) of the $\mathrm{W}$ removal process. 


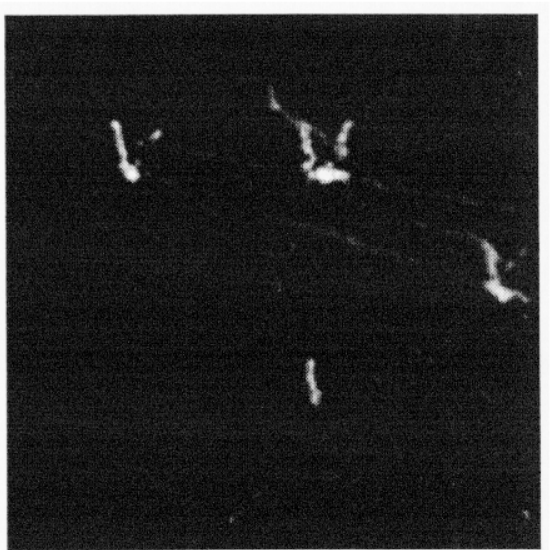

(a)

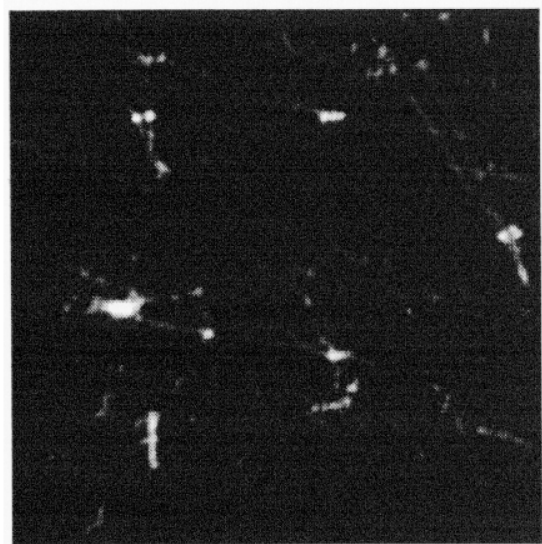

(b)

Figure 5. The positive (a) and negative (b) difference images of the $\mathrm{Al}$ and Ti features, plus residual $\mathrm{W}$ features for ART reconstructions with subtraction of the projections of the modified ideal model produced by using the iterative $\mathrm{W}$ removal method for the $-50^{\circ}$ to $+50^{\circ}$ case. These should be compared with the ART reconstructions done without iterative W removal in Figure 3.

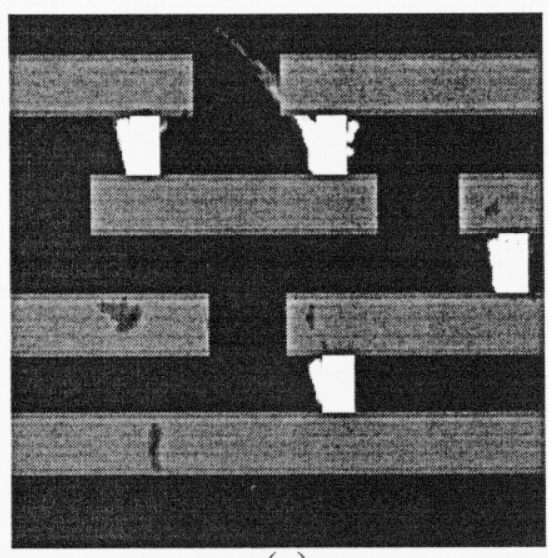

(a)

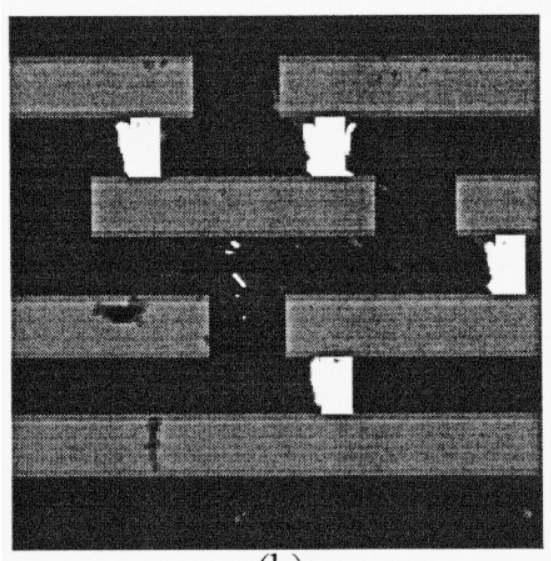

(b)

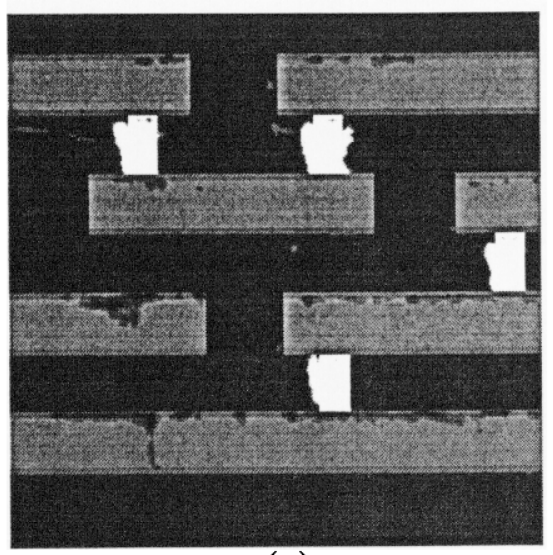

(c)

Figure 6. Final composite images produced by superimposing the reconstructed defects on the ideal model for the three angular ranges of (a) $-50^{\circ}$ to $+50^{\circ}$, (b) $-80^{\circ}$ to $+80^{\circ}$ and (c) $-90^{\circ}$ to $+90^{\circ}$ 


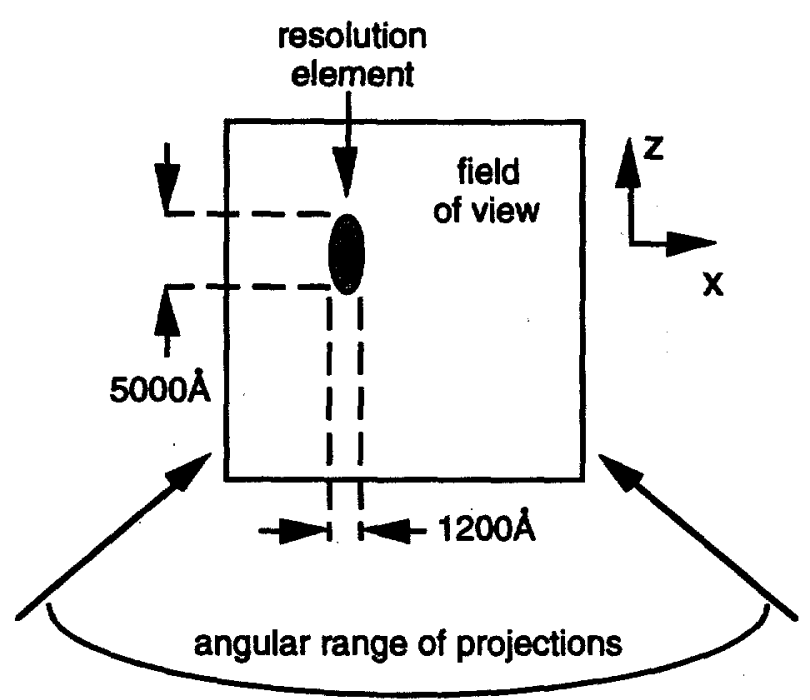

Figure 7. Estimated dimensions in 2-D of the resolution element within a reconstructed slice for the case of 21 projections over an angular range of $-50^{\circ}$ to $+50^{\circ}$.

\section{SUMMARY AND CONCLUSIONS}

We have shown that soft $x$-ray nanotomography could be used for evaluation and failure mode analysis of microchips. Realistic numerical simulations of the imaging process were performed based on an imaging system concept, and assuming the limited data case for which a small number of projections of the object over a restricted angular range are recorded.

Two numerical models of a single slice of a microchip were created for the simulations, one perfect, and one containing realistic, but acceptable deviations from the design as well as typical defects. The illuminating wavelength was taken to be $1.8 \mathrm{KeV}$ which is the point of maximum contrast between $\mathrm{SiO}_{2}$ and $\mathrm{Al}$. Artifacts due to the strong relative absorption of the W compared to everything else in the microchip are a significant problem.

A specialized approach to image reconstruction was devised specifically for $\mathrm{x}$-ray tomographic imaging of microchips with limited data. This reconstruction approach makes extensive use of prior knowledge of the microchip structure, and includes an iterative method for reducing the undesirable effects of the highly absorbing $\mathrm{W}$ features. The results show that submicron defects within a field of view of one cell in a microchip could be imaged in 3-D using such methods. This in turn could have an important impact on the development of new and better microchips.

\section{ACKNOWLEDGMENTS}

The authors would like to thank Steven J. Kirch of Intel Corporation's Materials Technology Department. We are also grateful to Ian McNulty and Wen-Bing Yun of the Advanced Photon Source for information on zone plates. This work was performed under the auspices of the U.S. Department of Energy under contracts W-7405-ENG-48 and W-31-109-ENG-38.

\section{REFERENCES}

1. See, for example, the Proceedings of the International Symposium on Testing and Failure Analysis (ISTFA), ASM International Pub.

2. See, for example, the Proceedings of the International Reliability Physics Symposium (IRPS), IEEE Pub.

3. W. S. Haddad, I. McNulty, J. E. Trebes, E. H. Anderson, R. A. Levesque and L. Yang, "Ultrahigh-Resolution X-ray Tomography", Science 266, pp. 1213-1215, (1994).

4. I. McNulty, W. S. Haddad, J. E. Trebes, E. H. Anderson, "Soft X-ray Scanning Microtomography with Submicrometer Resolution", Rev. Sci. Instrum. 66 (2), pp. 1431-1433 (1995).

* by Lawrence Livermore National Laboratory 
5. W. S. Haddad, J. E. Trebes, D. M. Goodman, H-R. Lee, I. McNulty, E. H. Anderson, A. O. Zalensky, "Ultra High Resolution Soft X-Ray Tomography", X-Ray Microbeam Technology and Applications, Wenbng Yun, Ed., Vol. 2516, pp. 102-107, (SPIE, San Diego, 1995).

6. U. Bonse, R. Nushardt, F. Busch, J. H. Kinney, R. A. Saroyan and M. C. Nichols, "X-Ray Tomographic Microscopy", X-Ray Microscopy III, A. Michette, G. Morrison, C. Buckley, Eds. (Springer-Verlag, Berlin, 1991).

7. J. H. Kinney, Q. C. Johnson, R. A. Saroyan, M. C. Nichols, U. Bonse, R. Nushardt and R. Pahl, Rev. Sci. Instrum. 59, 196 (1988).

8. P. Kirkpatrick and A. V. Baez, J. Opt. Soc. Am. 38, 766 (1948),

9. A. A. Krasnoperova, Z. Chen, F. Cerrina, E. Difabrizio, M. Gentili, W. Yun, B. P. Lai, E. S. Gluskin, "Microfocusing Optics for Hard X-Rays Fabricated by X-Ray Lithography", X-Ray Microbeam Technology and Applications, Wenbng Yun, Ed., Vol. 2516, pp. 102-107, (SPIE, San Diego, 1995).

10. C. Jacobsen, J. Kirz and S. Williams, Ultramicroscopy 47, 55-79 (1992).

11. B. L. Henke, P. Lee, T. J. Tanaka, R. L. Shimabukuro and B. K. Fujikawa, "Low-Energy X-Ray Interaction Coefficients: Photoabsorption, Scattering and Reflection", At. Data Nucl. Data Tables 27, 1 (1982).

12. A. C. Kak and M. Slaney, Principles of Computerized Tomographic Imaging chap. 3 (IEEE Press, New York, 1988). 


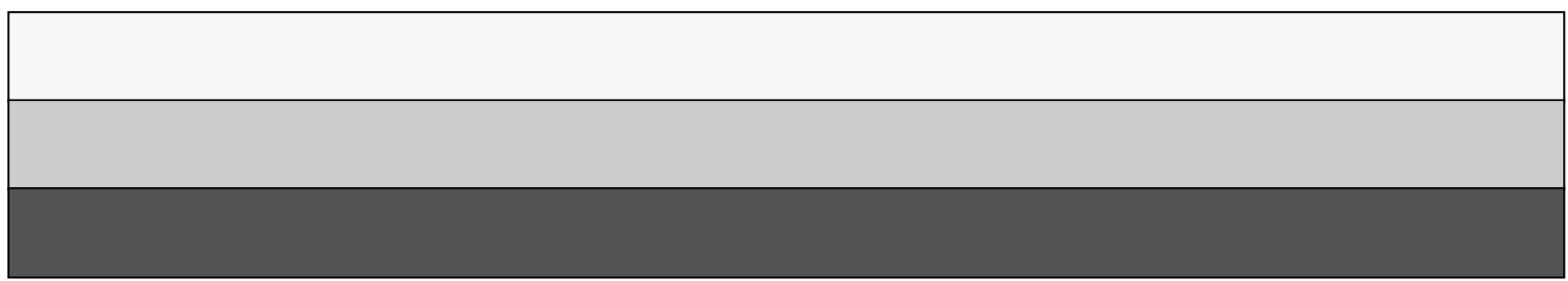

